\title{
Taking a Full Snapshot of Cancer Biology: Deciphering the Tumor Microenvironment for Effective Cancer Therapy in the Oncology Clinic
}

\author{
Kevin Dzobo ${ }^{1,2}$
}

\begin{abstract}
A bottleneck that is hindering therapeutics innovation in cancers is the current lack of integration of what we have learned in tumor biology as well as the tumor microenvironment (TME). This is because tumors are complex tissues composed of cancer cells, stromal cells, and the extracellular matrix (ECM). Although genetic alterations might cause the initial uncontrolled growth, resistance to apoptosis in cancer cells and stromal cells play additional key roles within the TME and thus influence tumor initiation, progression, therapy resistance, and metastasis. Therapies targeting cancer cells are usually insufficient when the stromal component of the TME causes therapy resistance. For innovation in cancer treatment and to take a full snapshot of cancer biology, anticancer drug design must, therefore, target both cancer cells and the stromal component. This expert review critically examines the TME components such as cancer-associated fibroblasts and ECM that can be reprogrammed to create a tumor-suppressive environment, thereby aiding in tumor treatment. Better cancer experimental models that mimic the TME such as tumor spheroids, microfluidics, three dimensional (3D) bioprinted models, and organoids will allow deeper investigations of the TME complexity and can lead to the translation of basic tumor biology to effective cancer treatments. Ultimately, innovative cancer treatments and, by extension, improvement in cancer patients' outcomes will emerge from combinatorial drug development strategies targeting both cancer cells and stromal components of the TME. Combinatorial treatment strategies can take the form of chemotherapy and radiotherapy (targeting tumor cells and stromal components) and immunotherapy that is able to regulate immune responses against tumor cells. This expert review thus addresses a previously neglected knowledge gap in cancer drug design and development by broadening the focus in cancer biology to TME so as to empower disruptive health care innovations in the oncology clinic.
\end{abstract}

Keywords: cancer research, drug design and development, tumor microenvironment, drug resistance, biomarkers, health care innovation

\section{Introduction}

$\mathbf{T}$ HERE HAVE BEEN SUBSTANTIVE ADVANCES in our understanding of cancer initiation and progression over the years. Cancer research has tended to focus on the cancer cells since time immemorial, but for a complete picture or snapshot of cancer biology, we also ought to understand the broader tumor microenvironment (TME) in which cancer cells reside and situate. Indeed, our understanding of the TME or its lack thereof remains one area hampering the development of effective cancer therapies able to produce durable cancer treatment. Recent studies advocate for the targeting of TME components.

This expert review critically examines the TME components such as cancer-associated fibroblasts (CAFs) and extracellular matrix (ECM) that can be reprogrammed to create a tumor-suppressive environment, thereby aiding in tumor treatment. Better cancer experimental models that mimic the TME such as tumor spheroids, microfluidics, three dimensional (3D) bioprinted models, and organoids will allow deeper investigations of the TME complexity and can lead to the translation of basic tumor biology to

\footnotetext{
${ }^{1}$ International Centre for Genetic Engineering and Biotechnology (ICGEB), Cape Town Component, Cape Town, South Africa.

${ }^{2}$ Division of Medical Biochemistry and Institute of Infectious Disease and Molecular Medicine, Department of Integrative Biomedical Sciences, Faculty of Health Sciences, University of Cape Town, Cape Town, South Africa.
} 
effective cancer treatments (Clevers, 2016; Drost and Clevers, 2018; Dzobo et al., 2018a, 2019a).

\section{Tumor Microenvironment}

The TME primarily comprises stromal cells and the ECM, among other types of cells. TME provides the cancer cells with the cues and physical environment to progress and eventually metastasize. Most cancer research and funding programs have focused mainly on genetic and some on epigenetic mechanisms of cancer initiation and progression, and the involvement of certain stromal cells such as CAFs, cancerassociated macrophages (CAMs) and cancer-associated neutrophils (CANs) cells (Senthebane et al., 2017). Besides the need to understand the source of these stromal cells, elucidating their phenotypic and functional heterogeneity of TME is key to designing strategies to target them as molecular leads in cancer therapeutics.

\section{Deciphering the TME}

CAFs have been shown to be the principal builders of the TME through their secretion of several factors and synthesis of the ECM that influences tumor growth and resistance to treatment (Kalluri, 2016; Senthebane et al., 2017, 2018). During tumor development, the stroma in general and CAFs specifically coevolve with cancer cells and continue to influence cancer cell behavior up to metastasis. It is important to note that inhibition and promotion of cancer progression have been reported for the stromal component or CAFs, with most studies indicating tumor promotion. Both CAFs and CAMs do synthesize ECM components and, therefore, affect the rigidity and elasticity, ultimately influencing stromal cell infiltration into tumors as well as drug availability (Provenzano et al., 2012; Saggar et al., 2013; Senthebane et al., 2018).

Recent study using chimeric antigen receptor $\mathrm{T}$ cell targeting fibroblast activation protein (FAP)-positive CAFs has demonstrated that CAFs are definitely involved in tumor growth and therapy resistance (Chung et al., 2014; Kakarla et al., 2013; Loeffler et al., 2006; Roberts et al., 2013; Zalcman et al., 2016). Although the ablation of subsets of CAFs arrested tumor growth, in some cases it has resulted in toxicity, cachexia, and anemia (Lo et al., 2015; Loeffler et al., 2006; Roberts et al., 2013; Wang et al., 2014). This is attributed to nonselective targeting of cells expressing markers such as FAP.

Several other cells including mesenchymal stem cells (MSCs) are known to express these markers. Furthermore, CAF subsets with tumor suppressive properties have been identified, complicating their targeting (Rhim et al., 2014). The development of effective therapies targeting stromal cells such as CAFs requires deeper insights into their phenotypic and functional heterogeneity. Several recent studies bring about timely knowledge and provide key insights into the role played by stromal cells in tumor growth, metastasis, and therapy resistance.

Senthebane and colleagues probed the origin of CAFs and demonstrated that CAFs can originate from MSCs that are reprogrammed by cancer cells through the action of transforming growth factor-beta (TGF- $\beta$ ) (Senthebane et al., 2017). Thus, infiltration of MSCs into the TME provides cells that can be transformed into CAFs. The infiltrated MSCs coevolve with cancer as the tumor evolves. Detailed analysis of cancer cell-stroma interaction within the TME will reveal other factors involved in the reprogramming of stromal cells.

Given their possible cellular sources, CAFs are phenotypically and functionally heterogeneous and different subsets have been described (Raz et al., 2018; Su et al., 2018). Su and colleagues described two surface markers, $\mathrm{CD} 10^{+}$and GPR $77^{+}$, that can be used to define a subset of CAFs that can be correlated with stemness, resistance to chemotherapy, as well as poor prognosis in breast and lung cancer (Su et al., 2018). These new markers together with canonical markers of CAFs such as smooth muscle actin-alpha (SMA- $\alpha$ ) and platelet-derived growth factor receptor-alpha (PDGFR- $\alpha$ ) will allow specific therapeutic targeting and possible ablation of subsets of CAFs with protumorigenic behavior in breast and lung cancer.

It is plausible that CAFs of different origins display differences in expression of certain markers, with both TME cues and characteristics of original cell type playing a huge part in this determination. New tumor models including the use of 3D organoids will help in delineating the interactions between stromal cells such as CAFs, CAMs, CANs, and others and cancer cells within the TME. Such work will help to translate optimism in TME research into clinical benefits.

In addition, Senthebane and coworkers demonstrated that functional interplay between the ECM and cancer cells influences cancer cell chemoresistance through provision of a physical barrier to drug delivery to cancer cells (Senthebane et al., 2018). The latter study revealed that ECM, and specifically both collagen and fibronectin, derived from CAFs and cancer cells, contributes to chemoresistance and increased migration of cancer cells in esophageal cancer (Senthebane et al., 2018). Gopal and coworkers described head and neck squamous cell CAF matrisome and identified fibronectin overexpression as an unfavorable prognostic marker (Gopal et al., 2017). Studies on the interaction between the ECM and cancer cells shall reveal physical tumorstroma interactions that are dependent on adhesion dynamics and will provide insights into tumor cell invasion, migration, and ultimately metastasis in the future.

\section{Road Ahead}

To illustrate the difficulties of working on CAFs, several studies employing animal models and sequencing techniques have revealed the presence of many subsets with specific functions and phenotypes (Cremasco et al., 2018; Erdogan and Webb, 2017; Hanley et al., 2016; Ishii et al., 2016). These studies have led to the identification of novel surface markers for subsets of CAFs in different cancers (Cremasco et al., 2018; Hanley et al., 2016; Ishii et al., 2016; Pietras et al., 2008; Raz et al., 2018). Beyond phenotypic characterization, functional heterogeneity displayed by CAFs subsets requires reclassification of some CAFs subsets. With new knowledge pointing to the primary tumor determining and influencing premetastatic distant tissues and organs, CAFs subsets at primary and at distant metastases must be determined.

The dynamic state of CAFs, from being tumor suppressive to tumor promoting through the action of tumor-derived factors, has been shown (Senthebane et al., 2017). Factors secreted by tumor cells and involved in attracting and 
transforming stromal cells into protumorigenic CAFs must be identified. Tumor-secreted factors including TGF- $\beta$ will allow the understanding of transformation and conversion of stromal cells into tumor-promoting cells such as CAFs (Raz et al., 2018; Senthebane et al., 2017). In addition, identification of signaling cascades activated by these tumor-derived factors remains important (Dzobo et al., 2019c).

Understanding the dynamic states of tumor-supporting cells such as CAFs and the ECM is crucial to revert such TME components to tumor-suppressive states. To this end, several receptor and signaling inhibitors are currently under trials for use in reverting CAFs into tumor-suppressive cells in different cancers (Froeling et al., 2011; Schnittert et al., 2018; Sherman et al., 2014).

Lastly, several research studies have shown the presence of CAF subsets in different cancers that display phenotypic and functional heterogeneity. These CAFs subsets are key to cancer treatment as they play several roles in the TME. These important roles range from recruiting stromal cells and other distant cells such as MSCs to the tumor, immune evasion, and suppression within the TME, ECM synthesis, angiogenesis, and chemoresistance (Dzobo et al., 2012, 2014, 2016).

Although it is very appealing on first glance, successful development of novel stroma-directed therapeutic strategies requires a deeper knowledge of the tumor-stroma interaction. As tumor cells have been shown to display heterogeneity, so does stromal cells such as CAFs (Dzobo et al., 2018b). Novel techniques including single cell analysis will be helpful in this regard (Dzobo et al., 2019b).

The importance of many studies on tumor-stroma interactions is that there is suggestion that stroma-directed therapeutic strategies will be of combinatorial nature, with drugs targeting both tumor cells and stromal cells. Furthermore, the characterization of the stromal or CAFs' influence on tumor progression and therapy response is key to management of patients' cancer. To this end, CAFs markers and more specifically markers of CAFs subsets must be well defined. Monitoring of these CAFs or stromal markers through liquid biopsy can be used as a link to poor prognosis or to monitor therapy response. Overall, stroma-directed therapies are poised to transform the theory and clinical practices in oncology toward integrative biology and holistic treatments, and novel drug development paradigms that address the complete picture in cancer biology.

\section{Cancer as a Metaphor}

Solid tumors can be viewed as organs or ecosystems/entities comprising cancer cells and stromal components. These entities are self-sustaining and do not respond to growth inhibition (Egeblad et al., 2010). The stromal components of tumors play key roles in the initiation and progression of the tumor. Borrowing from the social science field of global affairs, solid tumors can be viewed as "rogue regimes" in a somewhat peaceful world. Akin to targeted sanctions, depriving cancer cells/tumors of needed/necessary support or nutrients by targeting cancer stem cells (CSCs) and cancer cells and/or their subsets, their enablers such as CAFs, CAMs, CANs, tumor-associated blood vessels (TABVs), and ECM can cripple the whole rogue system, leading to its demise. That is the idea, theoretically.

The ultimate question is whether these "targeted sanctions" have been working or can work in cancer treatment?
Real life might teach us a thing or two. Has targeted sanctions led to the fall or pacification of "rogue regimes" anywhere in the world? And turning the table from societal context to biology, could cancer cells be eradicated or be induced into dormancy, perhaps akin to pacifying or better yet, democratizing "rogue regimes"? Time shall prove the true tests for cancer biology, but being cognizant of the prospects and challenges of TME is key to diagnostic and therapeutic innovation in oncology and cancer biology.

Although there may be successful stories to tell, evidence/ data showing that cancer treatment (chemotherapy, radiotherapy, immunotherapy) itself and the involvement of tumor stromal components can result in resistance is discouraging. This is akin to unsuccessful attempts at removing rogue regimes and can cause the hardening of such regimes. Although chemotherapy is effective treatment for cancers such as breast cancer, recent data demonstrate that chemotherapy promotes resistance and metastatic behavior through induction of mutations in cancer cells (Lønning and Knappskog, 2013) and the release of extracellular vesicles with prometastatic abilities, respectively (Keklikoglou et al., 2019).

A putative cancer treatment strategy can, through systematically converting members of the "rogue regimes" (CSCs, cancer cells) and their enablers (CAFs, CAMs, CANs, ECM, TABVs, ECM etc.) turn enemies into allies. Mirroring real-life global diplomacy, the re-educating of cancer cells, CAFs, CAMs, and CANs have been proposed as a way to treat cancer (Gok Yavuz et al., 2019; Shen et al., 2016). Controlling the composition of the ECM is another possible strategy (Senthebane et al., 2018). In cancers following the CSC model (Dzobo et al., 2016), does the mere removal of CSCs/founder cells (head of government or leading officials) result in effective cure or shrinkage of the tumor (collapse of rogue system)? Global affairs have shown that this does not always result in the intended result/outcome.

One thing is true. Successful cancer treatment, at least solid tumors, mirrors global diplomacy when dealing with "rogue regimes" (tumors). Tumors, like "rogue regimes," are different and require specific strategies for successful and effective treatment (Dzobo et al., 2018b). Our everyday lives and global diplomacy can teach us a lot about cancer treatment and possible successful strategies that are game changing and sustainable.

\section{Author Disclosure Statement}

The author declares there are no conflicting financial interests.

\section{Funding Information}

No funding was received for this article.

\section{References}

Chung K-M, Hsu S-C, Chu Y-R, et al. (2014). Fibroblast activation protein (FAP) is essential for the migration of bone marrow mesenchymal stem cells through RhoA activation. PLoS One 9, e88772.

Clevers H. (2016). Modeling development and disease with organoids. Cell 165, 1586-1597.

Cremasco V, Astarita JL, Grauel AL, et al. (2018). FAP delineates heterogeneous and functionally divergent stromal 
cells in immune-excluded breast tumors. Cancer Immunol Res 6, 1472-1485.

Drost J, and Clevers H. (2018). Organoids in cancer research. Nat Rev Cancer 18, 407-418.

Dzobo K, Leaner VD, and Parker MI. (2012). Feedback regulation of the alpha2(1) collagen gene via the Mek-Erk signaling pathway. IUBMB Life 64, 87-98.

Dzobo K, Leaner VD, and Parker MI. (2014). Absence of feedback regulation in the synthesis of COL1A1. Life Sci 103, 25-33.

Dzobo K, Motaung K, and Adesida A. (2019a). Recent trends in decellularized extracellular matrix bioinks for 3D printing: An updated review. Int J Mol Sci 20, 4628.

Dzobo K, Rowe A, Senthebane DA, Almazyadi MaM, Patten V, and Parker MI. (2018a). Three-dimensional organoids in cancer research: The search for the holy grail of preclinical cancer modeling. OMICS 22, 733-748.

Dzobo K, Senthebane DA, Rowe A, et al. (2016). Cancer stem cell hypothesis for therapeutic innovation in clinical oncology? Taking the root out, not chopping the leaf. OMICS 20, 681-691.

Dzobo K, Senthebane DA, Thomford NE, Rowe A, Dandara C, and Parker MI. (2018b). Not everyone fits the mold: Intratumor and intertumor heterogeneity and innovative cancer drug design and development. OMICS 22, 17-34.

Dzobo K, Thomford NE, Rowe A, Senthebane DA, and Dandara C. (2019b). Chapter 5-Single-Cell Omics: Deciphering Tumor Clonal Architecture. In: Single-Cell Omics. Barh D, Azevedo V, eds. Academic Press, pp. 61-97. Cambridge, MA, USA.

Dzobo K, Thomford NE, and Senthebane DA. (2019c). Targeting the versatile $\mathrm{Wnt} /$ beta-catenin pathway in cancer biology and therapeutics: From concept to actionable strategy. OMICS 23, 517-538.

Egeblad M, Nakasone ES, and Werb Z. (2010). Tumors as organs: Complex tissues that interface with the entire organism. Dev Cell 18, 884-901.

Erdogan B, and Webb DJ. (2017). Cancer-associated fibroblasts modulate growth factor signaling and extracellular matrix remodeling to regulate tumor metastasis. Biochem Soc Trans 45, 229-236.

Froeling FE, Feig C, Chelala C, et al. (2011). Retinoic acidinduced pancreatic stellate cell quiescence reduces paracrine Wnt-beta-catenin signaling to slow tumor progression. Gastroenterology 141, 1486-1497, 1497.e1-e14.

Gok Yavuz B, Gunaydin G, Gedik ME, et al. (2019). Cancer associated fibroblasts sculpt tumour microenvironment by recruiting monocytes and inducing immunosuppressive PD1+ TAMs. Sci Rep 9, 3172.

Gopal S, Veracini L, Grall D, et al. (2017). Fibronectinguided migration of carcinoma collectives. Nat Commun 8 , 14105.

Hanley CJ, Noble F, Ward M, et al. (2016). A subset of myofibroblastic cancer-associated fibroblasts regulate collagen fiber elongation, which is prognostic in multiple cancers. Oncotarget 7, 6159-6174.

Ishii G, Ochiai A, and Neri S. (2016). Phenotypic and functional heterogeneity of cancer-associated fibroblast within the tumor microenvironment. Adv Drug Deliv Rev 99, 186-196.

Kakarla S, Chow KK, Mata M, et al. (2013). Antitumor effects of chimeric receptor engineered human $\mathrm{T}$ cells directed to tumor stroma. Mol Ther 21, 1611-1620.

Kalluri R. (2016). The biology and function of fibroblasts in cancer. Nat Rev Cancer 16, 582-598.
Keklikoglou I, Cianciaruso C, Güç E, et al. (2019). Chemotherapy elicits pro-metastatic extracellular vesicles in breast cancer models. Nat Cell Biol 21, 190-202.

Lo A, Wang L-CS, Scholler J, et al. (2015). Tumor-promoting desmoplasia is disrupted by depleting FAP-expressing stromal cells. Cancer Res 75, 2800-2810.

Loeffler M, Krüger JA, Niethammer AG, and Reisfeld RA. (2006). Targeting tumor-associated fibroblasts improves cancer chemotherapy by increasing intratumoral drug uptake. J Clin Invest 116, 1955-1962.

Lønning PE, and Knappskog S. (2013). Mapping genetic alterations causing chemoresistance in cancer: Identifying the roads by tracking the drivers. Oncogene 32, 53155330.

Pietras K, Pahler J, Bergers G, and Hanahan D. (2008). Functions of paracrine PDGF signaling in the proangiogenic tumor stroma revealed by pharmacological targeting. PLoS Med 5, e19.

Provenzano PP, Cuevas C, Chang AE, Goel VK, Von Hoff DD, and Hingorani SR. (2012). Enzymatic targeting of the stroma ablates physical barriers to treatment of pancreatic ductal adenocarcinoma. Cancer Cell 21, 418-429.

Raz Y, Cohen N, Shani O, et al. (2018). Bone marrow-derived fibroblasts are a functionally distinct stromal cell population in breast cancer. J Exp Med 215, 3075-3093.

Rhim AD, Oberstein PE, Thomas DH, et al. (2014). Stromal elements act to restrain, rather than support, pancreatic ductal adenocarcinoma. Cancer Cell 25, 735-747.

Roberts EW, Deonarine A, Jones JO, et al. (2013). Depletion of stromal cells expressing fibroblast activation protein $\alpha$ from skeletal muscle and bone marrow results in cachexia and anemia. J Exp Med 210, 1137-1151.

Saggar JK, Yu M, Tan Q, and Tannock IF. (2013). The tumor microenvironment and strategies to improve drug distribution. Front Oncol 3, 154.

Schnittert J, Heinrich MA, Kuninty PR, Storm G, and Prakash J. (2018). Reprogramming tumor stroma using an endogenous lipid lipoxin A4 to treat pancreatic cancer. Cancer Lett 420, 247-258.

Senthebane D, Jonker T, Rowe A, et al. (2018). The role of tumor microenvironment in chemoresistance: 3D extracellular matrices as accomplices. Int J Mol Sci 19, 2861.

Senthebane DA, Rowe A, Thomford NE, et al. (2017). The role of tumor microenvironment in chemoresistance: To survive, keep your enemies closer. Int J Mol Sci 18, 1586.

Shen H, Yu X, Yang F, et al. (2016). Reprogramming of normal fibroblasts into cancer-associated fibroblasts by miRNAsmediated CCL2/VEGFA signaling. PLoS Genet 12, e1006244.

Sherman MH, Yu RT, Engle DD, et al. (2014). Vitamin D receptor-mediated stromal reprogramming suppresses pancreatitis and enhances pancreatic cancer therapy. Cell 159, 80-93.

Su S, Chen J, Yao H, et al. (2018). CD10+ GPR77+ cancerassociated fibroblasts promote cancer formation and chemoresistance by sustaining cancer stemness. Cell 172, 841-856. e816.

Wang L-CS, Lo A, Scholler J, et al. (2014). Targeting fibroblast activation protein in tumor stroma with chimeric antigen receptor $\mathrm{T}$ cells can inhibit tumor growth and augment host immunity without severe toxicity. Cancer Immunol research 2, 154-166.

Zalcman G, Mazieres J, Margery J, et al. (2016). Bevacizumab for newly diagnosed pleural mesothelioma in the 
Mesothelioma Avastin Cisplatin Pemetrexed Study (MAPS): A randomised, controlled, open-label, phase 3 trial. Lancet $387,1405-1414$.

Address correspondence to:

Kevin Dzobo, PhD

International Centre for Genetic Engineering and Biotechnology (ICGEB)

Cape Town Component Wernher and Beit Building (South) Anzio Road Observatory Cape Town 7925 South Africa

E-mail: kdzobosnr@yahoo.com

$\begin{aligned} & \text { Abbreviations Used } \\ 3 \mathrm{D} & =\text { three dimensional } \\ \text { CAFs } & =\text { cancer-associated fibroblasts } \\ \text { CAMs } & =\text { cancer-associated macrophages } \\ \text { CANs } & =\text { cancer-associated neutrophils } \\ \text { CSCs } & =\text { cancer stem cells } \\ \text { ECM } & =\text { extracellular matrix } \\ \text { FAP } & =\text { fibroblast activation protein } \\ \text { MSCs } & =\text { mesenchymal stem cells } \\ \text { PDGFR- } \alpha & =\text { platelet-derived growth factor receptor-alpha } \\ \text { SMA- } \alpha & =\text { smooth muscle actin-alpha } \\ \text { TABVs } & =\text { tumor-associated blood vessels } \\ \text { TGF- } \beta & =\text { transforming growth factor-beta } \\ \text { TME } & =\text { tumor microenvironment }\end{aligned}$

